\title{
From voice to knowledge: A proposal for a voice annotation system to support collaborative engineering design processes
}

\author{
Jorge D. Camba \\ Purdue University \\ jdorribo@purdue.edu
}

\author{
Ferrán Naya \\ Univ. Politècnica de \\ València, Spain \\ fernasan@upv.es
}

\author{
David Pérez-López \\ Univ. Politècnica de \\ València, Spain \\ dapelo@i3b.upv.es
}

\author{
Manuel Contero \\ Univ. Politècnica de \\ València, Spain \\ mcontero@upv.es
}

\begin{abstract}
This paper describes a novel voice interaction mechanism for capturing and managing design knowledge within a collaborative Computer-Aided Design (CAD) environment. We present a software module for speech recognition that integrates with a CAD application to allow the automatic creation of textual annotations in a $3 D$ model directly from voice data. Audio is transcribed automatically, resulting in a textual note that is searchable and available to other users via a Product Data Management (PDM) system, providing an intuitive mechanism to document modeling processes and design knowledge. The system consists of three functional blocks: (1) audio recording, (2) speech recognition, and (3) query management against a cloud-based service. In this paper, we justify the need for our system from a human-computer interaction standpoint and discuss the rationale of its design and implementation in the context of collaborative design communication. Finally, we discuss some application spaces that demonstrate the capability of voice annotations for capturing knowledge.
\end{abstract}

\section{Introduction}

Modern engineering design is an informationintensive activity where stakeholders create, share and exchange large amounts of information throughout the various stages of the collaborative design process. In many cases, this information is unstructured, taking the form of facts, suggestions, informal conversations, discussions, and opinions. Many decisions are communicated verbally, but the knowledge (at least a significant part of it) is often not captured or archived for future use because the process is time consuming, inefficient, and not cost effective [1].
Organizations that manage explicit and tacit knowledge in their processes usually follow a threestage approach: knowledge creation, knowledge retention and knowledge reuse [2]. In a product development context, design rationale is a key aspect of product knowledge. Design rationale is intended to represent information about design decisions (i.e. why decisions were made) as well as the relationships or dependencies that may either link decisions to the product representation or to other decisions [3]. Rationale capture, retrieval and feedback is an example of this three-stage approach.

A relevant concept related to design rationale is design intent, which is generally understood as a model's anticipated behavior once it undergoes alteration [4]. Here, the term "model" refers to the 3D $\mathrm{CAD}$ representation used in the product definition.

A number of mechanisms have been proposed to capture and record design intent and rationale. However, rarely have these tools been implemented successfully in industrial settings [5], partly due to the inherent inefficiencies at record taking, documentation and organization and sometimes because of a lack of motivation and incentives. In this regard, it is well known that designers are often reluctant to spend extra time documenting their designs with rationale [3]. Some fields, however, have a long tradition and history of success. In software engineering, for example, source code comments play an important role in code maintenance, documentation and reuse [6].

Considering the importance of design intent and rationale in collaborative design activities as well as recent advances in speech recognition and voice capturing technology, this paper proposes voice as an authoring user interface for 3D annotations to capture knowledge in engineering design scenarios and describes a software infrastructure that can support it. To this end, a voice transcription module was developed to enhance an existing 3D annotation system [7]. The module manages voice annotations and makes them searchable within a PDM system, 
which is used to provide knowledge retention and reuse capabilities. The ability to collect and process design knowledge from voice provides a novel mechanism for CAD that is particularly useful in scenarios where alternative inputs (e.g. keyboard, mouse, gesture-based interaction, etc.) may not be effective, such as in virtual reality environments, or when knowledge may emerge from interactive discussions or teleconferences where users can collaboratively engage to provide feedback without writing formal documentation. By keeping this data connected to the $3 \mathrm{D}$ model and searchable through the PDM system, a much richer product representation can be provided, and a more accurate record of the design process can be maintained.

In the next section, we discuss some relevant work to provide context and justify the need for our research. Section 3 gives a general overview of the annotation platform previously developed by the authors that was used to implement the voice annotation system. In sections 4 and 5, we discuss the architecture, design, and implementation details of the new voice module. Several applications spaces of our system are described in section 6. Conclusions and future work are presented in section 7 .

\section{Related work}

One of the earliest and most influential approaches in design rationale research is the IssueBased Information System (IBIS) proposed by Kunz and Rittel [8], which many researchers have used as a basis for developing their own solutions [5, 9]. Several variations and alternative approaches to IBIS have also been proposed, including the Procedural Hierarchy of Issues model (PHI) [10], the Question, Option, Criteria (QOC) [11] and the Issue Solution Artifact Layers (ISAL) [12].

There are two main approaches for implementing design rationale systems: automatic capture and user intervention. Both approaches are challenging, particularly in the case of true automation. Systems that require user intervention have achieved limited success, as they are typically considered too intrusive by designers and engineers. [13]. Design intent has received significantly less attention from the research community, as it is generally assumed that the 3D CAD models incorporate design intent in an implicit manner.

Some authors have applied storytelling and video sharing functionality to capture and share knowledge during the product development process [14]. Results have shown potential, although it is not clear whether this approach would function in an actual industrial setting. Similarly, other authors have compared annotations, word mappings and multiple-domain matrices to support communication between designers and engineers. Of the three tools, annotation was considered the most intuitive, visual and useful to provide a global overview of the information. [15].

The use of annotations as carriers of design and engineering knowledge has been empowered with new developments in Model-Based Definition (MBD) standards and Product Manufacturing Information (PMI) tools. For example, several authors have suggested the use of 3D annotations to enrich CAD models with design information [16-19]. This approach takes advantage of the global availability of product information across the enterprise as well as the functionality of PDM systems to share this information between the different stakeholders that participate in the development process. Additionally, this strategy relies on the designer to document the model in a manner that is similar to how source code is documented in the software engineering domain.

The use of annotations has proven successful as a method to make critical modeling decisions and design rationale available to other users by linking textual information to part geometry (text notes or hyperlinks to other documents or web sites). However, various limitations exist with the interaction mechanisms that are employed to create and maintain the 3D annotations in the model. Textual representation is effective for computer consumption, (e.g. applying data mining techniques to the annotated text) [20], but knowledge in an unstructured textual form can be cumbersome for humans, particularly when compared to verbal communication. Some efforts have been made to automate the creation of these annotations [21], but the results and the knowledge conveyed are not comparable to those provided by actual designers and engineers.

It has been reported that audio annotations can lead to higher-level comments [22]. A study by Chalfonte et al., [23] that evaluated user preferences in communication using textual and voice annotations revealed that users considered voice more expressive than text. Certain aspects of verbal communication such as speed, tone, emphasis, or pitch are difficult or impossible to replicate with text. In addition, humans tend to speak faster than they can type, and verbal communication is less prone to errors. Verbal communication is also more convenient than its textual counterpart when using portable devices, as small screen sizes and keyboards make it difficult to work with lengthy textual content. 
Voice annotations have been extensively explored in various domains. For example, in medicine voice annotations have been used to record notes in teleconsultations [24] and as dictation systems for development of reports in areas like radiology, pathology, and endoscopy [25]. In education, voice annotation systems have been implemented for language training [26] as well as instructor feedback and peer-discussion [27].

Voice annotations have also been implemented to support hand-free industrial maintenance tasks [28]. Researchers Harmon et al. [29] successfully demonstrated their voice-based annotation system for data visualization and architectural walkthroughs. Although voice annotations in this system could be linked to external textual annotations, the system did not implement speech-to-text capabilities [29]. In the context of design, the KnoCap tool [30], for example, was developed to collect design knowledge from conversations. However, this knowledge is not linked to the geometry of the 3D product representation.

Voice annotations are particularly useful in situations when users are unable to type or when typing becomes cumbersome or ineffective such as in virtual reality environments. In these scenarios, users can dictate their annotations, either by pointing at a specific element of the geometry or in response to another annotation.

In this paper, we propose a system for capturing design and engineering knowledge in a CAD model directly though voice interaction. The information becomes automatically linked to specific parts of the model and fully searchable and integrated within the PDM system.

\section{Annotation system}

The voice interaction system presented in this paper builds on the software architecture previously developed by the authors to manage extended annotations in CAD models [7]. The architecture provides a framework in the context of a Product Data Management (PDM) system for integrating Product and Manufacturing Information (PMI) into a 3D model directly from the CAD environment.

In our architecture, a dual representation is used to store 3D annotations. First, an internal data structure that works in conjunction with the PMI module of the CAD package serves both as a data repository within the $3 \mathrm{D}$ model and as a user interface to visualize and interact with the information directly from the geometry. Second, an external repository that is part of the PDM system is used to store additional metadata (including external references to other files) and make the content searchable. Both structures are automatically synchronized by a background process which is triggered every time an annotation is created, modified or deleted or when the model is saved to disk or uploaded to the PDM server.

The external repository is implemented as an XML file, which defines an "annotation" element for each annotation in the model. Annotation elements are described by the following child elements:

- text, content of the annotation in textual form.

- creator, name of the author of the annotation.

- date, date when the annotation was last modified.

- feature, name of the geometric element that the annotation is connected to.

- persistentId and internalld, unique identifiers created by the CAD package that connect the external and internal representations of the annotation.

- pos, 3D position of the annotation defined by its $\mathrm{x}, \mathrm{y}$ and $\mathrm{z}$ coordinates.

- website, URL or file name attached to the annotation.

- deleted, determines whether the annotation has been marked for removal.

- $\quad$ likes and dislikes, indicator of social impact that tracks the number of users that consider the annotation useful or not useful.

- annotationHistory, list of changes in the annotation over time.

The server side of the system provides various services to client applications such as PDM server authentication, item download (check-out, get version, and get revision), item upload (check-in, set revision, set lifecycle state, and lock/unlock data), and others. In our implementation, the PDM server uses Aras Innovator, but the architecture is modular enough to be easily implemented in other platforms.

The infrastructure that supports Aras Innovator includes a Microsoft's Internet Information (IIS) web server; a SQL database server for storage, indexing, and searching; and a file storage system where physical copies of the model are stored. The Aras data model is based on Items, which may have relationships to other Items. Our implementation uses (1) a directory Item, which organizes the models hierarchically and has relationships to $C A D$ Document Items; (2) a CAD Document Item, which stores the CAD model and the external annotations using File type relationships; and (3) an Annotation Item, which represents an annotation and includes all the annotations fields, a File type relationship that 


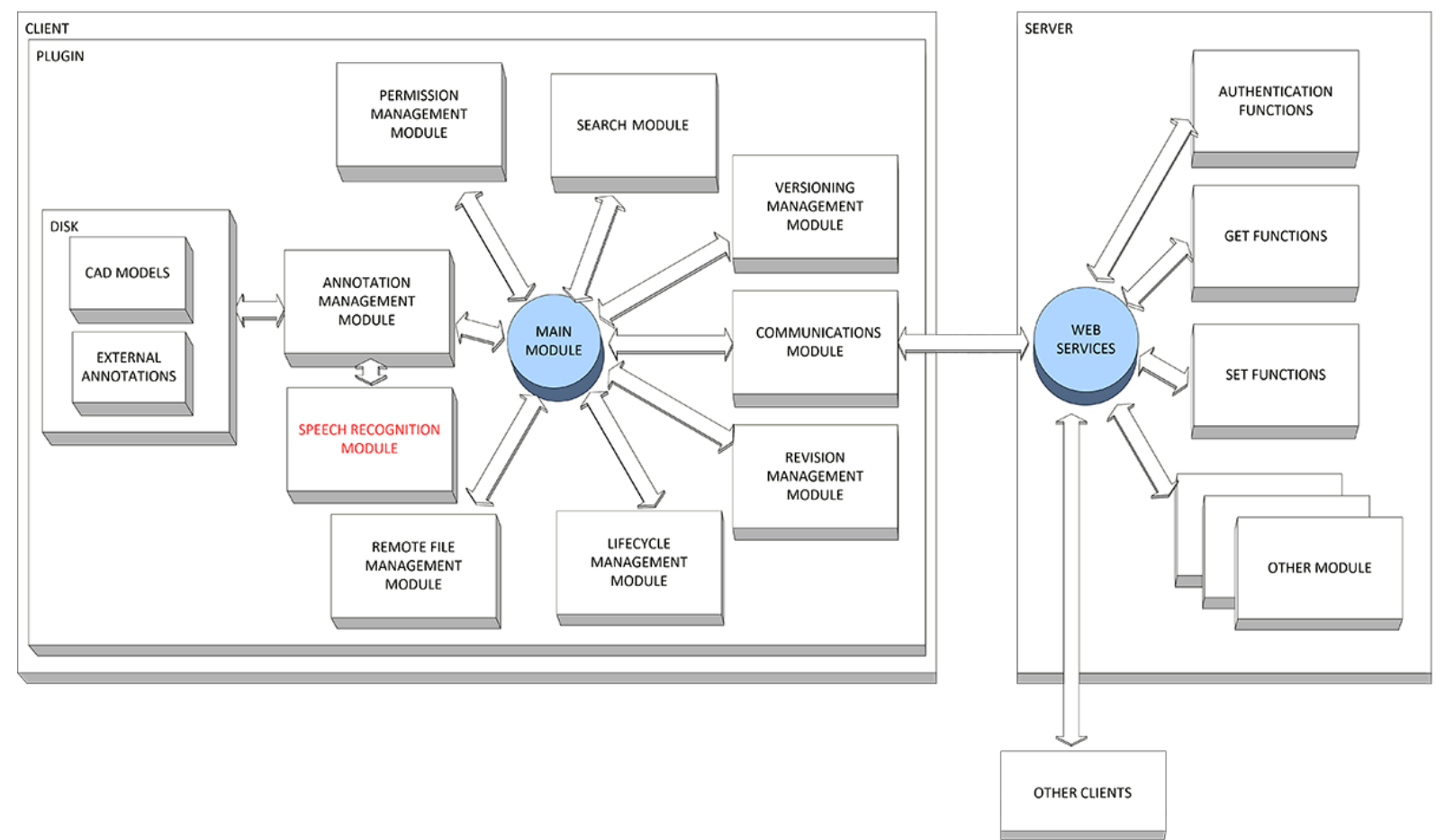

Figure 1. System architecture with speech recognition module

describes whether the annotation has an associated multimedia file, a second File type relationship that determines whether the annotation has an associated audio element, and a relationship with the $C A D$ Document Item where the model is stored.

The client can be understood as a set of modules that provide specific functionalities to a main module. While each module is responsible for performing a concrete task, the main module acts as a facilitator by sending and receiving data to and from the other modules. The modules and their functionalities are described in Table 1. The interactions are illustrated in Figure 1.

The client application is fully integrated with the CAD environment (e.g. SolidWorks, in our implementation) as an add-on or plugin. It was developed in Visual Basic as it is natively supported by the SolidWorks API.

The interface is organized in four tabs: Annotations, History, Browser, and Vault. The "Annotations" tab displays all the annotations in the model in tabular form where each annotation is shown on a separate row, as shown in Figure 2. The information displayed in the interface is linked to the annotations in the $\mathrm{CAD}$ model, i.e. selecting a row in the table automatically selects the corresponding annotation in the model. Basic functionality includes sorting annotations, searching, and filtering.
Table 1. Module descriptions

\begin{tabular}{|l|l|}
\hline Module & Description \\
\hline Annotation & $\begin{array}{l}\text { Controls model annotations and } \\
\text { synchronization operations } \\
\text { between the external and } \\
\text { internal representations }\end{array}$ \\
\hline Communications & $\begin{array}{l}\text { Controls interactions with the } \\
\text { PDM server }\end{array}$ \\
\hline $\begin{array}{l}\text { Permission } \\
\text { management }\end{array}$ & $\begin{array}{l}\text { Determines accessibility based } \\
\text { on user profiles. }\end{array}$ \\
\hline $\begin{array}{l}\text { Remote file } \\
\text { management }\end{array}$ & $\begin{array}{l}\text { Performs check-in and check- } \\
\text { out operations }\end{array}$ \\
\hline $\begin{array}{l}\text { Lifecycle } \\
\text { management }\end{array}$ & $\begin{array}{l}\text { Controls the document lifecycle } \\
\text { states }\end{array}$ \\
\hline $\begin{array}{l}\text { Revision } \\
\text { management }\end{array}$ & $\begin{array}{l}\text { Controls revisions and facilitates } \\
\text { the retrieval of earlier revisions }\end{array}$ \\
\hline Versioning & $\begin{array}{l}\text { Sets new versions and retrieves } \\
\text { previous ones }\end{array}$ \\
\hline Search & $\begin{array}{l}\text { Builds queries based on specific } \\
\text { search criteria }\end{array}$ \\
\hline $\begin{array}{l}\text { Speech } \\
\text { recognition }\end{array}$ & $\begin{array}{l}\text { Works with the Annotation } \\
\text { Manager to capture and transfer } \\
\text { speech to the remote recognition } \\
\text { engine and assign the } \\
\text { transcriptions to the annotations. }\end{array}$ \\
\hline
\end{tabular}




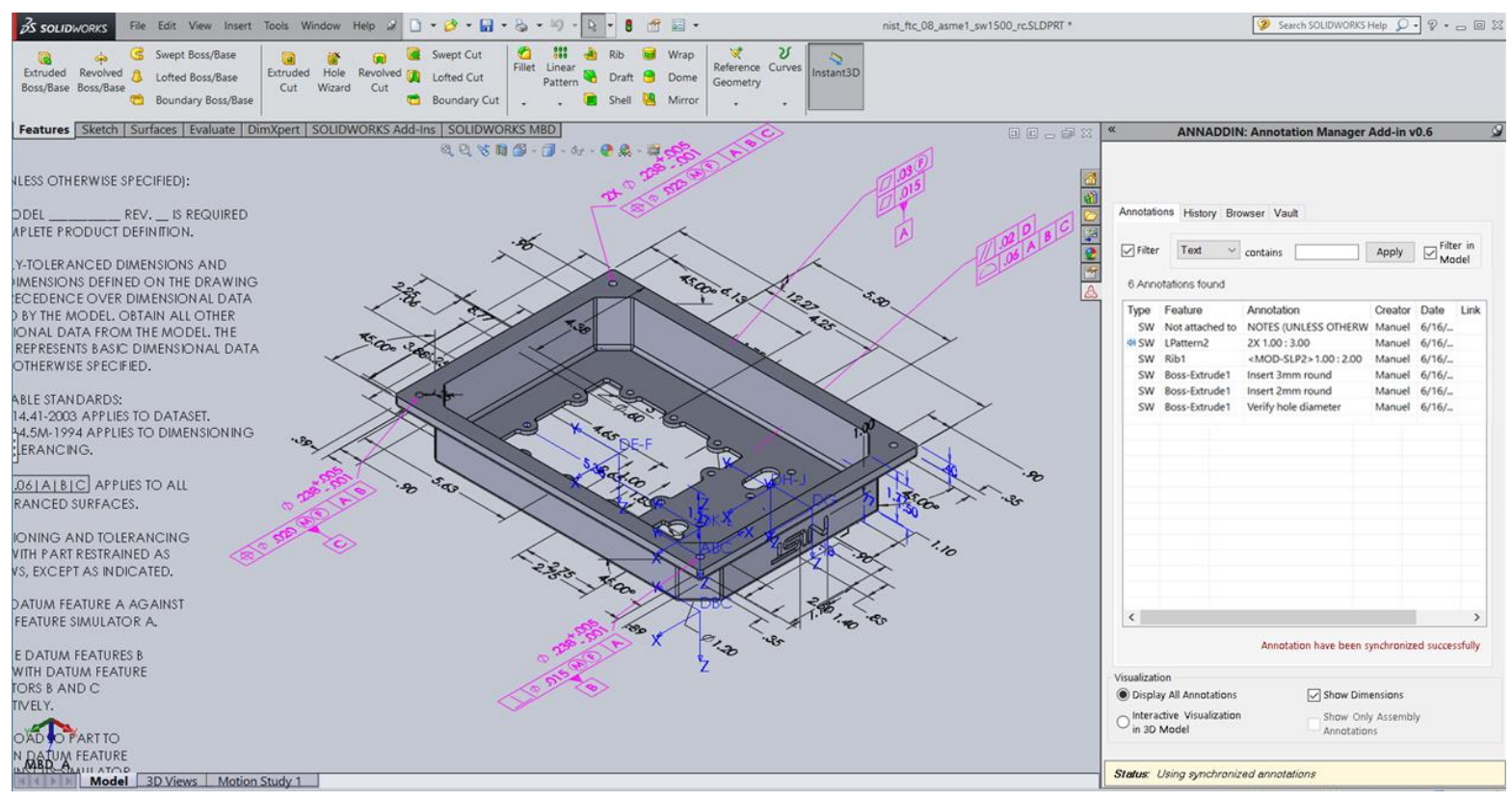

Figure 2: CAD environment with custom module interface (right) and annotations tab active [7]

The "History" tab provides a mechanism to track the history of every annotation associated to the model. This functionality is useful when performing security audits or analyzing the evolution of the model over time. An integrated web browser, a pdf file viewer and a video media player are available in the "Browser" tab where users can explore multimedia content associated with the annotations directly from the CAD environment. Finally, basic PDM functionalities (e.g. Check-In/Check-Out, change state, etc.) are available in the "Vault" tab. Authentication is required to connect to the Aras Innovator server and browse the repository based on the user's security profile. Contents are displayed in a directory view, as shown in Figure 3.

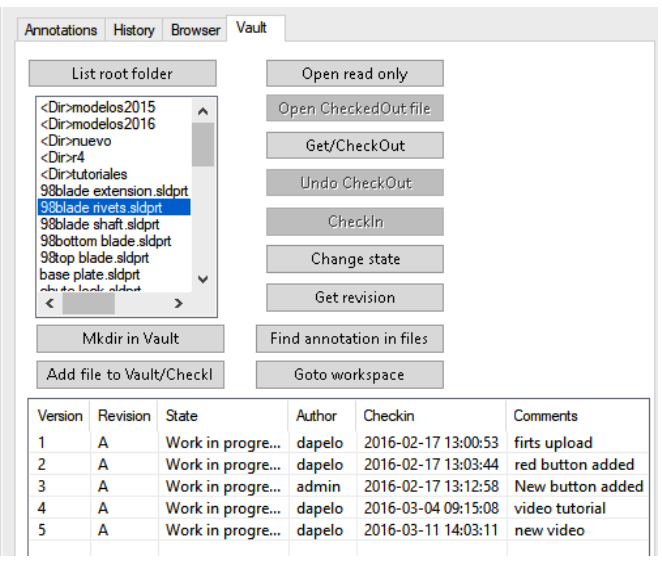

Figure 3. Vault tab. File selected and versioning information
The creation of annotations in the system is performed via mouse and keyboard interactions. The content of the annotation must be typed manually. In the next section, we describe the development of a new module that facilitates the creation of voicebased annotations and their automatic transcription.

\section{Speech recognition module design}

\subsection{Module requirements}

We present a module with speech recognition functionalities that facilitates the creation of voice annotations and the automatic transcription of the information. The module is based on a set of requirements identified as part of a human interaction study and are discussed below:

- The module must be able to create extended annotations from audio data and store them appropriately in the PDM server as well as within the 3D model.

- The module must be able to recognize speech and convert it to text in order to create a searchable extended annotation.

- The module must allow the filtering, searching and synchronization of the audio annotations and provide mechanisms to efficiently visualize and interact with the content (both as text and audio). 
- User interaction with the audio annotation must be available from the PDM server as well as from the CAD interface.

\subsection{Module architecture}

The audio and speech recognition functionalities were entirely implemented on the client side of our system (i.e. the CAD environment). It required the addition of a new child element, audioNote, to the general annotation element defined in the external XML file to store the name of the audio file.

The speech recognition module is composed of three functional blocks: (1) audio recording and playing, (2) speech recognition, and (3) query management. The block in charge of audio recording and playing was developed using NAudio [31]. Among other functionalities, this audio and MIDI library for .NET allows easy access to the microphone, the recording of audio files in different formats, and playing audio files from disk. When a voice annotation is to be created, the system starts recording audio from the microphone until the user manually stops the action or until a certain amount of time has passed (30 seconds, by default).

The second block of our audio system provides speech recognition capabilities based on the Google Speech to Text API [32]. This API can be used as Cloud Speech-to-Text client libraries or as Cloud Speech REST API. Although slightly harder to use, the official documentation recommends using the latter in production environments, as the client libraries are currently in alpha release and backwardcompatibility is not guaranteed.

The Word Error Rate (WER) was employed to evaluate the accuracy of the speech-to-text functionality. The Google Speech API is being constantly trained by millions of users on a daily basis and machine learning techniques are continuously improving. The WER metric depends on the language to recognize. As of 2017, the word accuracy of the Google API is estimated at $95 \%$ for U.S. English (4.9\% WER) [33], which makes it the first speech recognition framework to score below $5 \%$. Alternative tests $[34,35]$ have shown a higher WER ( $7.4 \%$ and $13.5 \%$, respectively).

The third block of the audio system is used to manually build queries against a REST service through the .NET HttpWebRequest class. The service requires authentication, which can be performed by OAuth, an open standard for token-based authentication, or an API key. In order to track data from different users and teams, the latter option was selected. Data interchange is accomplished through formatted JSON files. The module builds a JSON query with the audio data recorded by block 1 and sends it to the Google Cloud. Audio data is sent as a base64 encoded string, as shown in Figure 4. Next, a JSON response is sent back by the Google Cloud that contains the recognized text, as shown in Figure 5. The .NET library Newtonsoft.json [36] is used to manage the queries and responses. This library provides methods to convert JSON files to objects and vice versa, which helps the system to efficiently interchange data with the Google Cloud.

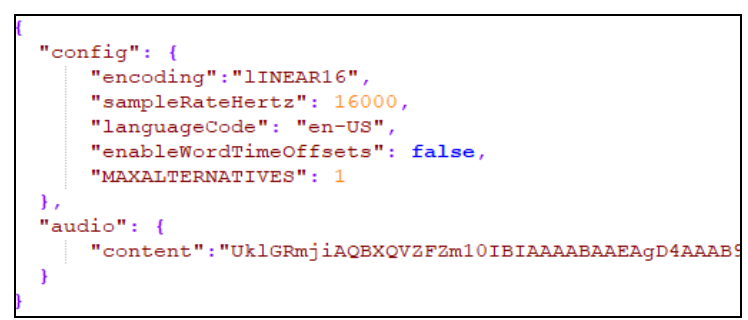

Figure 4. Sample JSON sent to Google Cloud

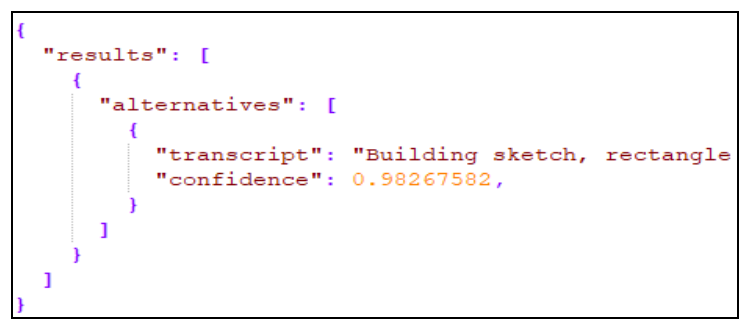

Figure 5. Sample JSON response from Google Cloud

Finally, the 3D model, the extended annotation file (XML), and all the multimedia files linked to the annotations are uploaded to the PDM. The system works offline, and changes are synchronized when a PDM connection is available. The architecture of the module is shown in Figure 6.

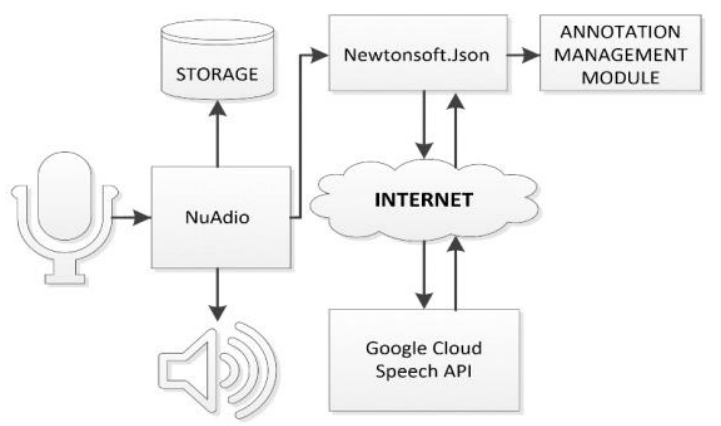

Figure 6. Speech recognition flow diagram

\section{Interface}

Users can create or edit a voice annotation by selecting "Create voice annotation" from the 
contextual menu available in the annotation manager interface, as shown in Figure 7. A dialog box prompts the user to select the language (a default language can be set at the application level). It should be noted that Google Cloud Speech API provides automatic language recognition, but the functionality is still in beta phase. When "Create voice annotation" is selected, a new recording starts. A voice spectrum animation that reacts to the user's voice acts as a visual indicator that a recording is in progress. The recording can be stopped manually by clicking the "Stop recording" button, or automatically after 30 seconds (to avoid recording long periods of silence or generating extremely large files).

When the recording stops, a local data file containing the audio is sent to the Google Cloud. The service returns the transcribed speech in textual form. This text and the local audio file are then connected to the annotation. Visually, in the user interface, voice annotations are represented with a triangular shape (to mimic the traditional "play" button) followed by the transcribed text, as shown in Figure 8. Similarly, in the annotations table, a speaker icon is used to represent a voice annotation (see Figure 9). A "Play Annotation" command is available to play audio annotations from the CAD environment.

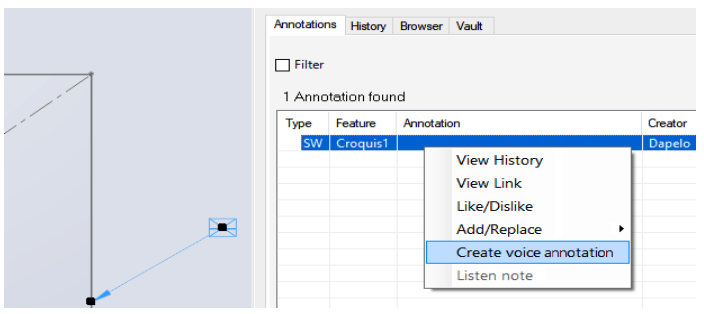

Figure 7. Creating a voice annotation

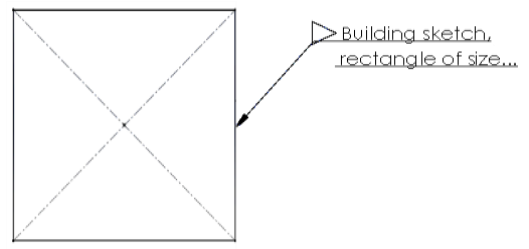

Figure 8. Symbol for a voice annotation with transcribed text attached to a profile

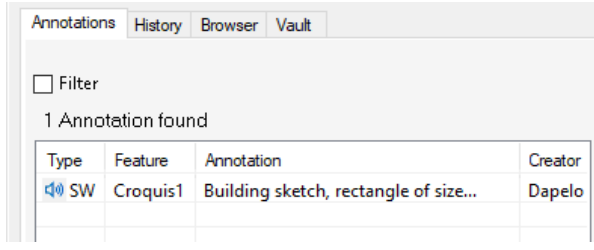

Figure 9. Visual indicator of a voice annotation in the annotations table

\section{Application space}

We present potential applications that demonstrate the capability of voice-based annotations and the knowledge capturing techniques of our system.

\subsection{Collaborative design review sessions}

The natural and intuitive interaction styles enabled by our voice-based annotation system are particularly useful in multi-user scenarios that require extensive collaboration such as collaborative design reviews. In situations that involve design and model review meetings with different specialists (sometimes in geographically dispersed teams) in real time, large amounts of data are often generated through team discussions, comments, feedback and numerous explanations. These data may refer to design alternatives, product's aesthetics such as textures and colors, ergonomics, manufacturing, or design and structural issues arising from analysis and simulation results, to name a few. Data often revolve around specific aspects of the $3 \mathrm{D}$ CAD model, which is shared in real time among all the stakeholders.

Design review sessions usually involve users pointing at various parts of the geometry, selecting, moving and rotating components of an assembly, or marking and highlighting errors that need to be fixed or features that need to be modified or redone. These actions are typically accompanied by lengthy discussions and/or descriptions (often by different people) and sometimes the data can be complex and multi-layered, with connections and references to other designs or models and even past discussions and previous meetings.

Capturing and documenting all the information generated during collaborative design review meetings is challenging. Design review software is common, but significant notetaking is required. When 3D models are annotated, only short notes are normally included which often lack the necessary detail and context to provide the design rationale. It is therefore difficult to comprehensively document the model. In these scenarios, voice-based annotations can streamline design review and model review workflows by providing a pervasive interface that can capture information, make it searchable and immediately available in the PDM system through 3D model, and automatically structure and connect this information to specific product attributes and properties. Furthermore, this type of voice annotations may also facilitate interactions in design review sessions conducted in virtual environments. 


\subsection{Customer involvement and co-design}

As engineering and manufacturing firms transition to Industry 4.0 and Model-Based Enterprise paradigms, the reliance on customers' knowledge and experiences for product development, improvement and innovation will likely increase. Customers are gradually becoming co-creators and key resources for creating value, particularly in industrial products. These practices may lead to stronger ties with customers, higher market visibility and significant reductions in time to market. To enable the active dialog and communication necessary for success, integration within the company's supply chain and significant involvement of the customers throughout the product life cycle are required.

Customer involvement in the product development process can be implemented at various levels, from basic personalization and customization options to becoming fully involved in co-designing the product. In any case, utilizing our system, users can easily capture customer feedback and knowledge when a 3D model is used as a mediator in the communication process and instantly have the information available to other users through the PDM. The use of voice provides a natural and intuitive interface for users to participate in co-design activities and provide feedback and suggestions about a particular product. We envision these customer experiences to possibly be conducted through mobile devices such as phones and tablets, where users can visualize the $3 \mathrm{D}$ model and provide input during a real time co-design session.

\subsection{Document generation}

From a documentation standpoint, our voicebased annotations (with the corresponding transcriptions) can be used in combination with assembly models and interactive exploded views for the automatic creation of technical manuals and installation, maintenance, manufacturing, safety, and step-by-step assembly instructions. We envision CAD models with voice annotations that capture the operation descriptions, operating conditions, and operation flow of a product and intuitively convey work instructions, which can reduce the burden of preparing technical documentation and make the format and quality of these documents uniform.

In addition, by having the information linked to the 3D model, the content of this documentation becomes dynamic, which reduces the time and cost of future updates and ensures that the end documentation always contains the most up to date information. Finally, the end documentation and all the related processes are traceable in the PDM system. Re-use of existing content and processes is also possible.

\subsection{CAD training}

From an educational standpoint, our system provides valuable opportunities for assessment. In student-centered learning environments, providing personalized feedback to students is essential to give each student the insights they need to address specific gaps in their knowledge or abilities. To accomplish this, student feedback needs to be meaningful, detailed, timely and frequent. Personalized feedback is particularly important in online and distance education environments. However, instructors, especially those responsible for teaching large classes, are under increasing pressure to develop effective feedback, even though the time an instructor can devote per student is decreasing.

In an engineering design or 3D modeling class, many assignments involve high order cognitive activities, which can be challenging and time consuming to assess. Feedback for each assignment can be extensive and often difficult to elaborate without referencing the model or certain aspects of the student assignment. In many cases, feedback is facilitated through labor intensive manual procedures, even when computer-supported tools are available. For example, assessing the quality of a parametric 3D model requires navigating through the structure of the model, which can be comprised of dozens of items, opening and checking each one of these items, finding mistakes or inefficiencies, and determining the level of correctness based on a set of multi-dimensional criteria. Providing a detailed assessment of student performance can be a tedious and time-consuming activity.

Using our system, instructors can simplify the creation of feedback by selecting the specific aspects of the model that the student needs to address and giving a detailed verbal explanation of the issues. In addition, the expressiveness of voice annotations can also contribute to create a richer and more personalized connection with the student.

\section{Conclusions and future work}

In this paper, we brought effective voice-based interaction to knowledge capture, annotation, and design documentation processes. We introduced a framework built on a CAD environment where users can create knowledge by recording comments and discussions directly from the CAD environment. The 
information is then automatically transcribed and stored in a PDM system, where it becomes searchable, enabling analysis and data mining.

We introduced the design and implementation of a novel architecture which yields guidelines for the interaction and user experience design of voice-based mechanisms in collaborative design environments. The architecture was validated through the development of a software prototype.

We proposed application spaces to demonstrate how the voice-based annotations enabled by our system could be utilized to effectively communicate design intent and rationale in collaborative design sessions as well as to provide feedback in training. We believe that voice-based knowledge capture and management in collaborative design interfaces can expand product definition and documentation beyond geometry and basic annotations to convey richer information to users.

From a practical standpoint, the effectiveness of the proposed approach depends on the accuracy, precision, efficiency and reliability of the speech recognition module as well as the real-time characteristics of the transcription process. Similarly, voice annotations may not be sufficient to communicate certain design aspects that require users to illustrate or sketch concepts visually or represent time-based processes. In these cases, mark-up, selection, and sketching tools may be used in combination with voice annotations, which can add complexity to the corresponding data structures in the PDM system. As future work, we are interested in conducting a study with a large user group to measure the effectiveness of our approach when compared to traditional methods of design documentation and communication. In addition, possible technical improvements include the automatic recognition of users in collaborative sessions so the information can be automatically linked to the creators as they engage in discussions.

\section{Acknowledgements}

This work was supported by the Spanish grant DPI2017-84526-R (MINECO/AEI/FEDER, UE), project "CAL-MBE, Implementation and validation of a theoretical CAD quality model in a Model-Based Enterprise (MBE) context."

\section{References}

[1] N. Anerousis and E. Panagos, "Making voice knowledge pervasive" IEEE Pervasive Computing, 2002, 1(2), pp. $42-48$.
[2] N. Levallet and Y.E. Chan, "Knowledge loss and retention: the paradoxical role of IT", Successes and Failures of Knowledge Management, 2016, pp. 97-111, Morgan Kaufmann.

[3] S. Szykman, R.D. Sriram and W.C. Regli, "The role of knowledge in next-generation product development systems", Journal of computing and information Science in Engineering, 2001, 1(1), pp. 3-11.

[4] J. Otey, P. Company, M. Contero and J.D. Camba, "Revisiting the design intent concept in the context of mechanical CAD education", Computer-Aided Design and Applications, 2018, 15(1), pp. 47-60.

[5] R.H. Bracewell, S. Ahmed, and K.M. Wallace, "DRed and design folders: a way of capturing, storing and passing on knowledge generated during design projects" ASME 2004 International Design Engineering Technical Conferences and Computers and Information in Engineering Conference 2004 Jan 1, pp. 235-246.

[6] D. Steidl, B. Hummel and E. Juergens, "Quality analysis of source code comments", 201321 st International Conference on Program Comprehension (ICPC), pp. 83-92. IEEE.

[7] J.D. Camba, M. Contero, P. Company, and D. Pérez, "On the integration of model-based feature information in Product Lifecycle Management systems", Int. Journal of Information Management, 2017, 37(6), pp. 611-621.

[8] W. Kunz and H.W. Rittel, "Issues as elements of information systems" Berkeley, CA: Institute of Urban and Regional Development, University of California; 1970.

[9] J. Conklin, A. Selvin, S. Buckingham Shum, and M. Sierhuis, "Facilitated Hypertext for Collective Sensemaking: 15 Years on from gIBIS", Proceedings 12th ACM Conference on Hypertext and Hypermedia, Århus, Denmark, 2001.

[10] R.J. McCall, "PHI: a conceptual foundation for design hypermedia”, Design Studies, 1991, 12(1), pp. 30-41.

[11] A. MacLean, R.M. Young, V.M. Bellotti, and T.P. Moran, "Questions, options, and criteria: elements of design space analysis", Human-Computer Interaction, 1991, 6(3), pp. 201-250.

[12] Y. Liu, Y. Liang, C.K. Kwong, and W.B. Lee, "A new design rationale representation model for rationale mining" Journal of Computing and Information Science in Engineering, 2010, 10(3), 031009.

[13] S.K. Chandrasegaran, K. Ramani, R.D. Sriram, I. Horváth, A. Bernard, R.F. Harik and W. Gao, "The evolution, challenges, and future of knowledge representation in product design systems", Computer-Aided Design, 2013, 45(2), pp. 204-228. 
[14] J. Zammit, J. Gao, R. Evans, and P. Maropoulos, “A knowledge capturing and sharing framework for improving the testing processes in global product development using storytelling and video sharing", Proceedings of the Institution of Mechanical Engineers, Part B: Journal of Engineering Manufacture, 2018, 232(13), pp. 2286-2296.

[15] G. Rasoulifar, C. Eckert and G. Prudhomme, "Supporting communication between product designers and engineering designers in the design process of branded products: a comparison of three approaches", CoDesign, 2014, 10(2), pp. 135-152.

[16] Q.Y. Ma, L.H. Song, D.P. Xie and M.J. Zhou, "Development of CAD Model Annotation System Based on Design Intent" Applied Mechanics and Materials, 2017, 863, pp. 368-372, Trans Tech Publications.

[17] J. Camba, M. Contero, M. Johnson, and P. Company, "Extended 3D annotations as a new mechanism to explicitly communicate geometric design intent and increase CAD model reusability", Computer-Aided Design, 2014, 1(57), pp. 61-73

[18] M. Lundi, E. Lejon, A. Dagman, M. Näsström and P. Jeppsson, "Efficient design module capture and representation for product family reuse", Journal of Computing and Information Science in Engineering, 2017, 17(3), 031002-1 - 031002-16.

[19] S. Sandberg and M. Näsström, “A proposed method to preserve knowledge and information by use of knowledge enabled engineering", ASME 2007 International Design Engineering Technical Conferences and Computers and Information in Engineering Conference, pp. 207-212. American Society of Mechanical Engineers.

[20] Y. Yong, H. Deyu, W. Hong and Z. Gang, "Data mining and recommendation of engineering note items in MBD dataset", Proceedings of the 2019 11th International Conference on Computer and Automation Engineering, pp. 1-6. ACM.

[21] Y. Cheng, F. He, X. Lv and W. Cai, "On the role of generating textual description for design intent communication in feature-based 3D collaborative design", Advanced Engineering Informatics, 2019, 39, pp. 331-346.

[22] S.B. Cousins, M. Baldonado and A. Paepcke, "A systems view of annotations", Tech. Report 9910022, 2000.

[23] B.L. Chalfonte, R.S. Fish, and R.E Kraut, "Expressive richness: a comparison of speech and text as media for revision", Proceedings of the SIGCHI Conference on Human factors in computing systems, 1991, pp. 21-26.

[24] L. Czekierda, F. Malawski and P. Wyszkowski, "Holistic approach to design and implementation of a medical teleconsultation workspace", Journal of biomedical informatics, 2015, 57, 225-244.

[25] J. Vajpai and A. Bora, "Industrial Applications of Automatic Speech Recognition Systems", International Journal of Engineering Research and Applications, 2016, 6(3), 88-95.

[26] Y.Y. Lian, C.M. Chen and Y.R. Li, "Effects of collaborative multimedia annotations on elementary school students' vocabulary learning performance", Proceedings of the 6th IIAI International Congress on Advanced Applied Informatics (IIAI-AAI), 2017, pp. 649-654. IEEE.

[27] D. Yoon, N. Chen, B. Randles, A. Cheatle, et al., "RichReview++: deployment of a collaborative multimodal annotation system for instructor feedback and peer discussion", Proceedings of the 19th ACM Conference on Computer-Supported Cooperative Work \& Social Computing, 2016, pp. 195-205. ACM.

[28] X.S. Zheng, P. Matos da Silva, C. Foucault, S. Dasari, M. Yuan, and S. Goose, "Wearable solution for industrial maintenance", Proceedings of the 33rd Annual ACM Conference Extended Abstracts on Human Factors in Computing Systems, 2015, pp. 311-314. ACM.

[29] R. Harmon, W. Patterson, W. Ribarsky and J. Bolter, "The virtual annotation system", Proceedings of the IEEE Virtual Reality Annual International Symposium, 1996, pp. 239-245

[30] A.M. Soria and A. van der Hoek, "Collecting design knowledge through voice notes", Proceedings of the 12th International Workshop on Cooperative and Human Aspects of Software Engineering, pp. 33-36, 2019.

[31] M. Heath, "Audio and MIDI library for .NET", 2016. https://github.com/naudio/NAudio (Retrieved 02/13/2019)

[32] Google. Speech API - Speech Recognition, 2017. https://doi.org/10.2307/3094807

[33] N. van der Velde, "The Complete Guide to Speech Recognition Technology",

https://www.globalme.net/blog/the-present-future-ofspeech-recognition, (Retrieved 09/3/2019).

[34] L. Velikovich, I. Williams, J. Scheiner, P.S. Aleksic, P.J. Moreno and M. Riley, "Semantic Lattice Processing in Contextual Automatic Speech Recognition for Google Assistant”, Interspeech, 2018, pp. (2222-2226).

[35] O. Biran, "You Shall Not Speak: Benchmarking Famous Speech Recognition APIs for Chatbots" https://cai.tools.sap/blog/benchmarking-speechrecognition-api/, (Retrieved 09/3/2019).

[36] J. Newton-King, "Newtonsoft", 2012. https://www.newtonsoft.com/json (Retrieved 02/13/2019). 